\title{
Frontal theta EEG activity correlates negatively with the default mode network in resting state
}

\author{
René Scheeringa $^{\mathrm{a}, *}$, Marcel C.M. Bastiaansen ${ }^{\mathrm{a}, \mathrm{b}}$, Karl Magnus Petersson ${ }^{\mathrm{a}, \mathrm{b}}$, Robert Oostenveld ${ }^{\mathrm{a}}$, \\ David G. Norris ${ }^{\text {a }}$, Peter Hagoort ${ }^{\text {a,b }}$ \\ ${ }^{a}$ F.C. Donders Centre for Cognitive Neuroimaging, Radboud University Nijmegen, The Netherlands \\ ${ }^{\mathrm{b}}$ Max Planck Institute for Psycholinguistics, Nijmegen, The Netherlands
}

Received 28 February 2007; accepted 16 May 2007

Available online 12 July 2007

\begin{abstract}
We used simultaneously recorded EEG and fMRI to investigate in which areas the BOLD signal correlates with frontal theta power changes, while subjects were quietly lying resting in the scanner with their eyes open. To obtain a reliable estimate of frontal theta power we applied ICA on band-pass filtered (2-9 Hz) EEG data. For each subject we selected the component that best matched the mid-frontal scalp topography associated with the frontal theta rhythm. We applied a time-frequency analysis on this component and used the time course of the frequency bin with the highest overall power to form a regressor that modeled spontaneous fluctuations in frontal theta power. No significant positive BOLD correlations with this regressor were observed. Extensive negative correlations were observed in the areas that together form the default mode network. We conclude that frontal theta activity can be seen as an EEG index of default mode network activity.
\end{abstract}

(C) 2007 Elsevier B.V. All rights reserved.

Keywords: EEG; fMRI; Independent component analysis; Frontal theta; Default mode network; Resting state

\section{Introduction}

The simultaneous recording of EEG and fMRI signals has sharply increased in popularity over the last few years. While the initial efforts in this multimodal enterprise were aimed at solving technical problems stemming mainly from artifacts in the EEG generated in the MR environment (Allen et al., 2000; Allen et al., 1998; Bonmassar et al., 1999; Bonmassar et al., 2002), subsequent research has explored the relationship between EEG and BOLD from various vantage points. For instance, quite some effort has been devoted to identify BOLD correlates of epileptoform EEG activity (Aghakhani et al., 2004; Al-Asmi et al., 2003; Benar et al., 2006; Lemieux et al., 2001; Salek-Haddadi et al., 2002). More recently, simultaneous EEG/ fMRI has been successfully applied to investigate cognitive

\footnotetext{
* Corresponding author. FC Donders Centre for Cognitive Neuroimaging, Trigon building, PO Box 9101, 6500 HB Nijmegen, The Netherlands. Tel.: +31 243610 750; fax: +31243610989.

E-mail address: rene.scheeringa@fcdonders.ru.nl (R. Scheeringa).
}

phenomena such as performance monitoring (Debener et al., 2005) and sustained attention (Eichele et al., 2005). Apart from epilepsy research most studies have focused on the BOLD correlates of ongoing oscillatory activity during resting state (i.e., recorded while the subject was quietly lying in the scanner, with no explicit task), focusing on the posterior alpha rhythm and, to a lesser extent, the beta rhythm (Feige et al., 2005; Goldman et al., 2002; Goncalves et al., 2006; Laufs et al., 2006, 2003a,b; Moosmann et al., 2003). In these studies, the general idea has been that spontaneous fluctuations in the amplitude of a given oscillatory signal can be correlated with spontaneous fluctuations in the BOLD signal. It has been suggested that such BOLD correlates are indicative of functionally connected brain structures that are related to the rhythmic neuronal activity observed in the EEG.

Studies investigating the BOLD correlates of spontaneous fluctuations in alpha activity (Feige et al., 2005; Goldman et al., 2002; Goncalves et al., 2006; Laufs et al., 2006, 2003a,b; Moosmann et al., 2003) have shown that the expected posterior visual regions and also other regions across the cortex are 
negatively correlated with posterior alpha fluctuations. Recent findings suggest that there is considerable individual variation in areas that correlate with the alpha fluctuations and that these can be linked to differences in the total power spectrum of an individual (Goncalves et al., 2006; Laufs et al., 2006). Laufs et al. (2006) observed that negative alpha-BOLD correlations in posterior visual areas go together with relatively high theta power during alpha desynchronisation, while relative high beta power during an entire session is related to negative alphaBOLD correlations in regions thought to be related to attention in the parietal and frontal lobes. A few studies (Laufs et al., 2003b; Moosmann et al., 2003) have also reported BOLD correlates of beta activity. Where Moosmann et al. (2003) observed mainly negative beta-BOLD correlations, Laufs et al. (2003b) reported positive correlations in regions that show an overlap with the default mode network (Fox et al., 2005; Raichle et al., 2001).

An interesting new avenue in the analysis of resting state data is to investigate which brain regions correlate with the oscillatory components associated with more specific cognitive operations. An example of such a component is the frontal theta rhythm, which is well known from both EEG and MEG literature. This component has a frequency range of roughly $3-$ $8 \mathrm{~Hz}$, and is most prominent over midline fronto-central electrodes. Frontal theta has been observed during various cognitive activities that require attention or short term memory (Burgess and Gruzelier, 1997; Inanaga, 1998; Ishihara and Yoshii, 1972; Laukka et al., 1995; Lazarev, 1998; Smith et al., 1999), and is often studied during mental arithmetic (Asada et al., 1999; Burgess and Gruzelier, 1997; Inanaga, 1998; Inouye et al., 1994; Iramina et al., 1996; Ishihara and Yoshii, 1972; Ishii et al., 1999; Lazarev, 1998; Mizuki et al., 1980; Sasaki et al., 1996; Smith et al., 1999). More recently, frontal theta power has been found to increase with working memory load (Gevins et al., 1997; Jensen and Tesche, 2002; Krause et al., 2000; Onton et al., 2005), indicating a possible role of theta oscillations in working memory maintenance. Source modeling attempts have localized the frontal theta rhythm to the anterior cingulate or the medial frontal cortex (Asada et al., 1999; Gevins et al., 1997; Ishii et al., 1999; Onton et al., 2005). Interestingly, Mizuhara et al. (2004) as well as Sammer et al. $(2005,2007)$ demonstrated that frontal theta increases can be observed in the EEG acquired during fMRI. Both Sammer et al. (2007) and Mizuhara et al. (2004) report positive correlations between BOLD and theta in the insula, hippocampus, superior temporal cortex, cingulate cortex and frontal areas while subjects performed a mental arithmetic. Where Sammer et al. (in press) reported only positive correlations, Mizuhara et al. (2004) reported predominantly negative correlations between frontal theta and BOLD in medial frontal, posterior cingulate, temporal and inferior parietal areas.

The present study aims at delineating the BOLD correlates of spontaneous fluctuations in frontal theta power, in a simultaneous EEG/fMRI setting. Srinivasan et al. (2006) report that theta power is highest at frontal midline electrodes in resting state, indicating that the frontal theta rhythm is also detectable outside task conditions. Since most studies investigating the relation between oscillatory activity and the BOLD signal used the resting state paradigm, we decided to follow this line of research. To our knowledge this is also the first time the relationship between frontal theta and the BOLD signal is studied in conditions where subjects did not perform a task. To this end, we first identify the theta rhythm in the EEG during an eyes open, resting state recording session. Subsequently we correlate the theta power with the BOLD signal. Separation of the frontal theta rhythm from artifacts and other rhythms in the theta frequency range with different spatial distributions is accomplished by applying independent component analysis (ICA). Previous studies (Makeig et al., 2002; Onton et al., 2005) have demonstrated that ICA can successfully be applied to EEG data to separate frontal theta activity from other (oscillatory) activity. Debener et al. $(2005,2006)$ demonstrated that ICA can be applied to EEG data measured in the MRI scanner to obtain single trial measures of evoked potentials that can be successfully correlated with the BOLD signal. Similarly, by correlating power fluctuations of the frontal theta components obtained by ICA with the BOLD signal in resting state, we hope to gain some insight into the neuronal structures that are associated with the frontal theta rhythm in humans.

\section{Methods}

\subsection{Subjects}

Twenty right handed volunteers (17 female, 3 male, age range: 18-28) participated in the study after giving written informed consent. None had a neurological impairment, experienced neurological trauma or had used neuroleptics. The subjects were paid a small fee for their participation.

\subsection{Design and procedure}

Subjects came to the F.C. Donders Centre 1 hour before the scanning session started. First the electrode cap was applied and an instruction for a working memory task was given. While in the scanner, the subjects first participated in a working memory experiment for approximately 1 hour, divided in three blocks. After this experiment a resting state measurement was carried out in which subjects were asked to watch a black fixation cross presented on a grey background for $10 \mathrm{~min}$. At the end of the scanning sessions a T1 weighted anatomical MRI was acquired. This anatomical scan is not used in the analysis of the fMRI data presented in this paper. Between measurements there were small breaks of a few minutes. Subjects were also allowed to go outside the scanner during these breaks. Only the data from the resting state session is used in the analysis presented here.

\subsection{Electrophysiological recordings}

EEG was recorded at 29 scalp sites (Fp1, Fp2, F3, F4, C3, C4, P3, P4, O1, O2, F7, F8, T7, T8, P7, P8, Fz, Cz, Pz, FC1, FC2, CP1, CP2, FC5, FC6, CP5, CP6, TP9, TP10) with a MR-compatible BrainAmp MR amplifier (Brainproducts, Munich, Germany) and an MR-compatible electrode cap 


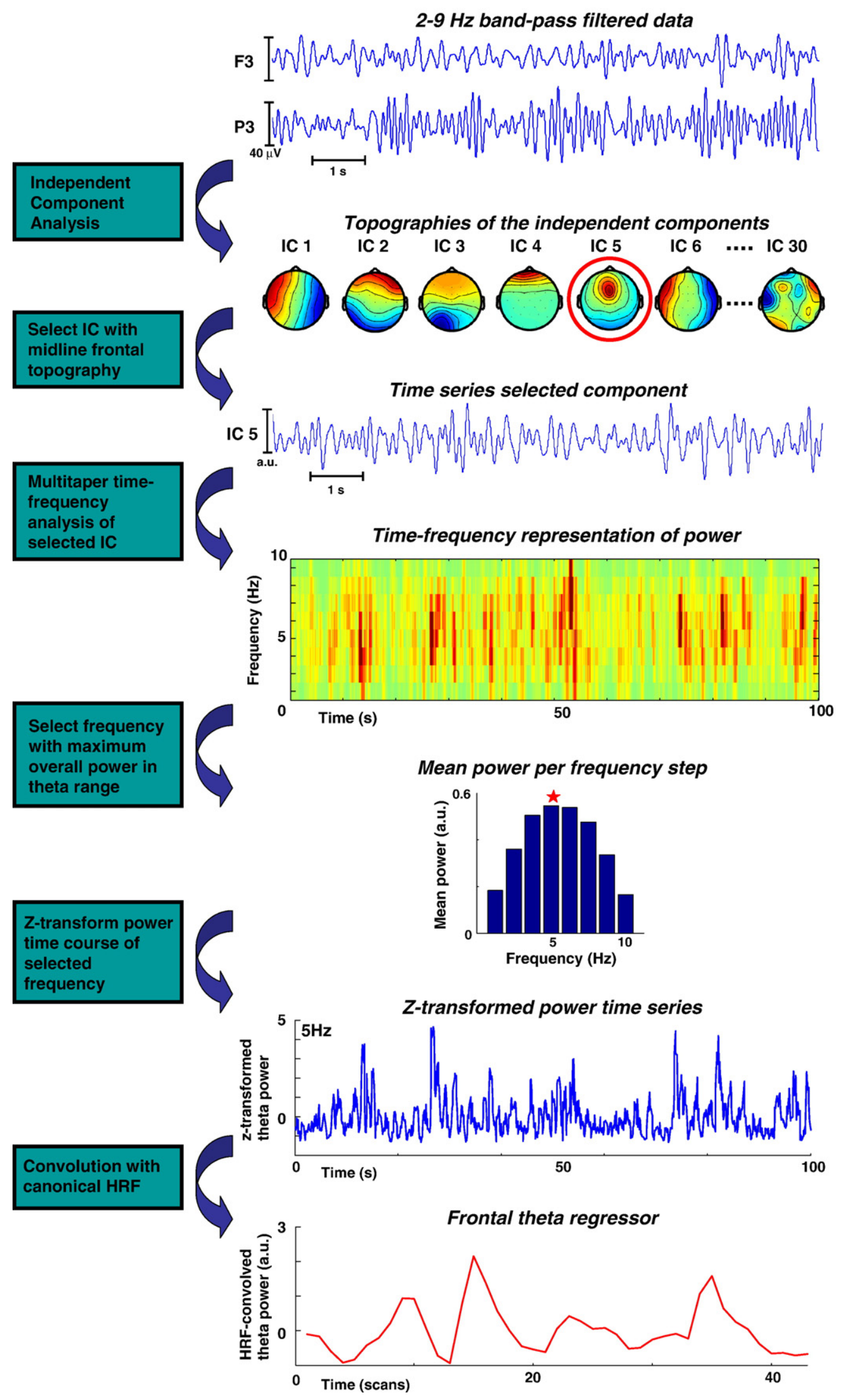

Fig. 1. Schematic representation of the different steps in the construction of the regressor modeling fluctuations in frontal theta activity starting at the 2-9 Hz band-pass filtered data. This procedure is in more detail described in the Methods section. 
equipped with carbon wired sintered $\mathrm{Ag} / \mathrm{AgCl}$ electrodes (Easycap, Herrsching-Breitbrunn, Germany). The reference electrode was located at FCz. To record the vertical EOG one electrode was placed under the right eye. The ECG was measured by two dedicated electrodes attached to the electrode cap. One electrode was placed on the sternum, the other electrode was placed on the clavicle, near the shoulder. A $250-\mathrm{Hz}$ hardware filter was placed between the electrode cap and the amplifier. The EEG was recorded with a $0.16 \mathrm{~s}$ time constant and a $100 \mathrm{~Hz}$ low-pass filter and continuously sampled at $5 \mathrm{kHz}$. Impedances were kept under $5 \mathrm{k} \Omega$. All recordings were done with Brain Vision Recorder software (Brainproducts).

\subsection{Image acquisition}

MRI measurements were performed using a 1.5 T Sonata whole body scanner (Siemens, Erlangen, Germany). Functional images were acquired using a gradient echo EPI sequence (TR $2.34 \mathrm{~s}$ including $50 \mathrm{~ms}$ dead time; FOV $=224 \mathrm{~mm}, \mathrm{TE}=30 \mathrm{~ms}$,
33 slices, $3.0 \mathrm{~mm}$ slice-thickness with $0.5 \mathrm{~mm}$ slice-gap; voxelsize $3.5 \times 3.5 \times 3.0 \mathrm{~mm}$ ).

\subsection{MRI artifact removal}

The EEG data were corrected for gradient and pulse artifacts along the lines described by Allen et al. (1998, 2000) using Vision Analyzer (Brainproducts). A 20-volume, baseline corrected sliding average was used for the correction of the gradient artifacts. In order to achieve this, 10 extra volumes were recorded before and after the $10 \mathrm{~min}$ of data used for analysis. After gradient correction the data were low-pass filtered at $100 \mathrm{~Hz}$ and down sampled to $500 \mathrm{~Hz}$. The average pulse artifact was calculated based on a sliding average, time locked to the R-peak present in the bipolar derivation of the two ECG electrodes. This sliding average was scaled to an optimum least squares fit for each heart beat using the scaling option in Vision Analyzer before it was subtracted from the data. The data were subsequently rereferenced to a common average reference. The original reference channel was recomputed as FCz.

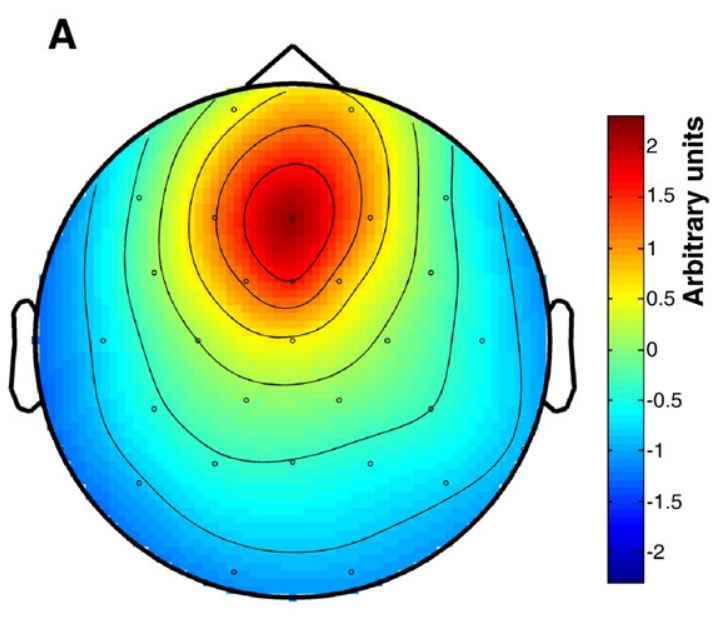

B

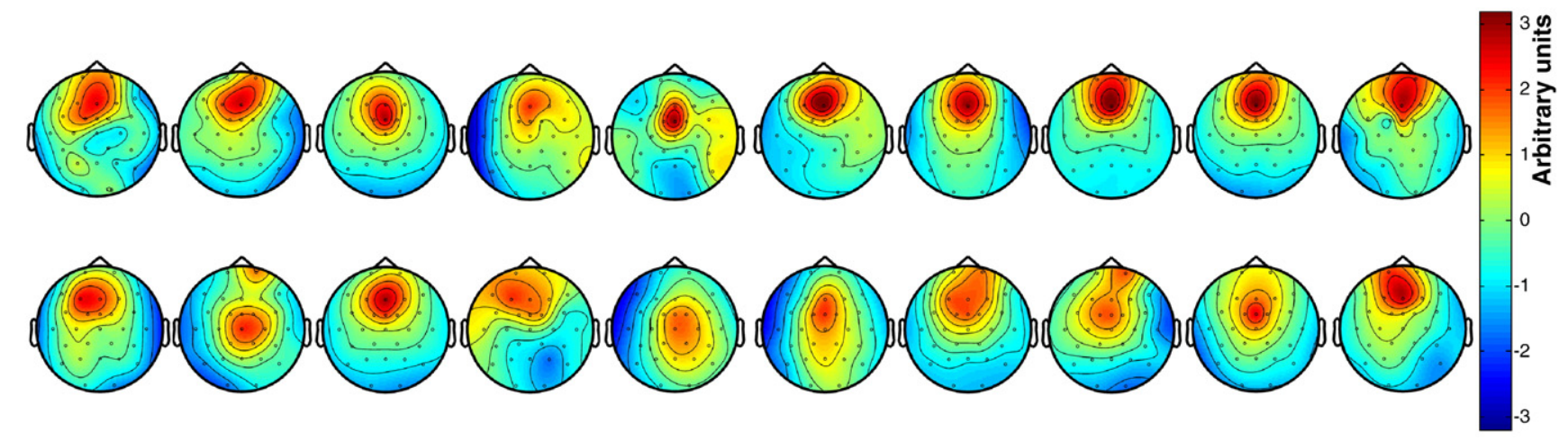

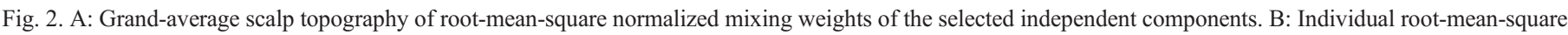

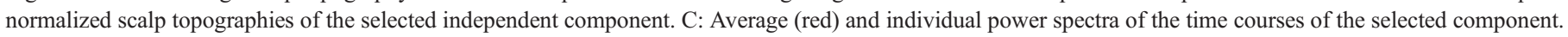
Individual spectra are normalized to the average power in the $2-9 \mathrm{~Hz}$ range. 
A

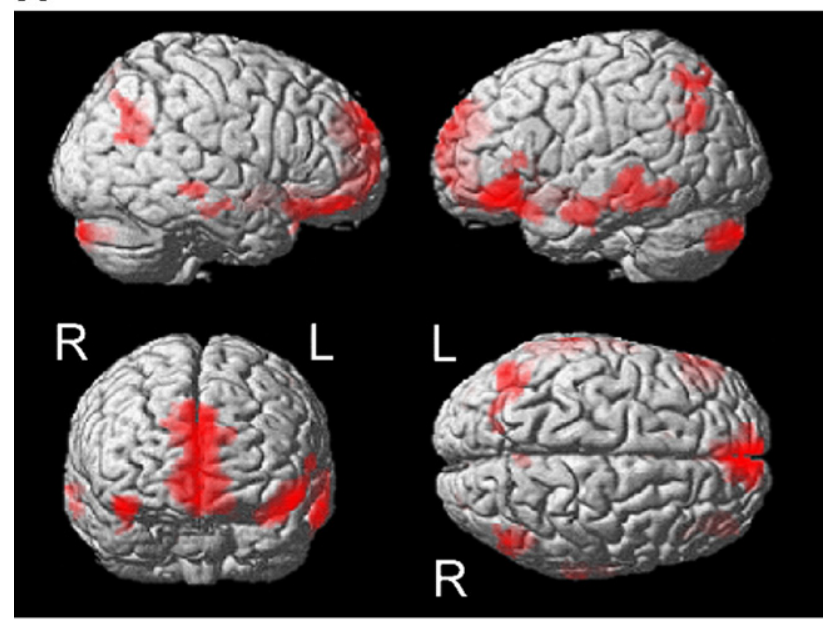

B

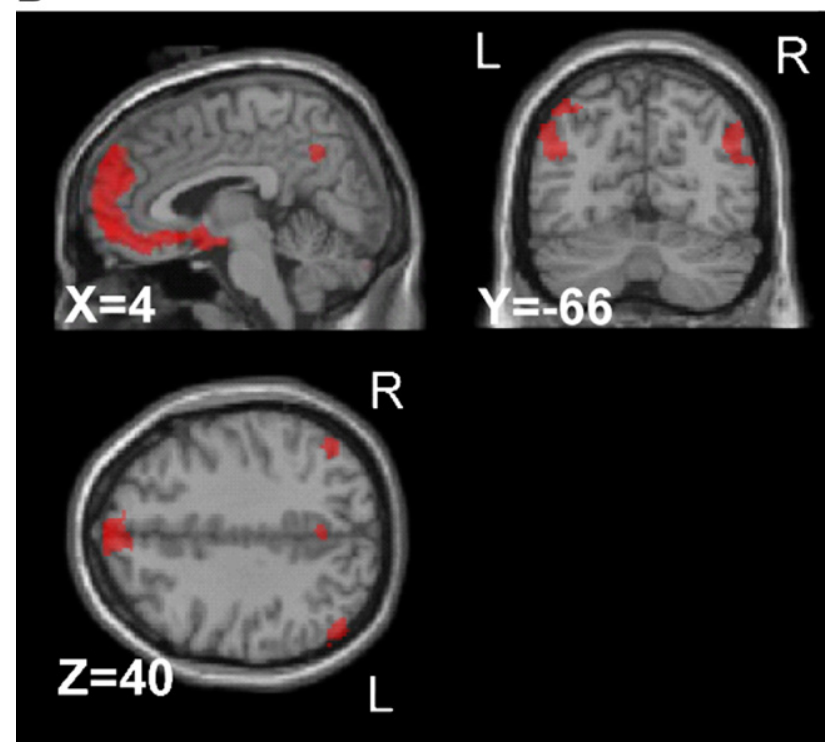

Fig. 3. Significant negative correlations with frontal theta power rendered on a standard MNI brain (A) and in saggital, transversal and coronal slices (B).

\subsection{Regressor construction}

Since we were specifically interested in frontal theta activity, and the recorded EEG is a mixture of artifacts and different theta sources, we applied infomax independent component analysis (ICA) (Bell and Sejnowski, 1995) on the entire $10 \mathrm{~min}$ of resting state data to obtain a 'clean' frontal theta estimate.

For this analysis a weight change of $10^{-7}$ was used as a stop criterion with the maximum number of iterations being 512 . This proved to be sufficient to obtain a stable solution for the data from all subjects. To remove the residual gradient artifacts and low frequency noise as much as possible before applying ICA, a 2-9 Hz band-pass filter was applied to the data. ICA was carried out in the Fieldtrip toolbox for EEG/MEG-analysis (FC Donders Centre for Cognitive Neuroimaging, Nijmegen, The Netherlands; see http://www.ru.nl/fcdonders/fieldtrip), using the runica algorithm (Makeig et al., 1997) implemented in EEGLAB version 5.03 (Delorme and Makeig, 2004). Both Fieldtrip and EEGLAB run under matlab (MathWorks, Natick, MA).
For each subject, the component with a topography that showed a dipolar pattern that best matched the fronto-central scalp distribution of the clusters of theta components reported by Makeig et al. (2002) and Onton et al. (2005) was selected for further analysis. If more than one component with a fronto-central distribution was observed, the component accounting for the most variance was selected (4 subjects in total). A time-frequency analysis of power was applied on the time series of the selected component. For this we used a multi-taper approach (Mitra and Pesaran, 1999). Power was analyzed from 1.25 to $10 \mathrm{~Hz}$ in $1.25 \mathrm{~Hz}$ steps for every $100 \mathrm{~ms}$. Multi-tapering was performed with $800 \mathrm{~ms}$ time smoothing and $2.5 \mathrm{~Hz}$ frequency smoothing.

For each selected IC the time course of one frequency bin was used to form a regressor. This was done by selecting from the frequency bins centered around $3.75,5$ and $6.25 \mathrm{~Hz}$ the one with the maximal power. The maximum was based on the average power in these frequency bins over the 10-minute recording session. The entire time series for the selected frequency bin was extracted to form the basis for the regressor. First this time series was $z$-transformed. In order to remove artifacts, time points with a higher absolute $z$-score than 5 were set at zero. The high $z$-score of 5 was chosen because of the skewed distribution of values. The resulting time series was again $z$-transformed before it was convolved with the canonical hemodynamic response function (HRF) implemented in SPM5 (Wellcome Department of Imaging Neuroscience, London, UK see http://www.fil.ion.ucl.ac.uk/spm), resulting in an EEGbased regressor that models spontaneous fluctuations in frontal theta power (for a schematic representation of the regressor construction see Fig. 1).

\section{7. $f M R I$ analysis}

Processing and analysis of the fMRI data were carried out in SPM5. The fMRI data was corrected for movements, corrected for differences in slice acquisition time, anatomically normalized

Table 1

Brain regions showing significant negative correlations with frontal theta power

\begin{tabular}{|c|c|c|c|c|c|c|}
\hline \multirow[t]{2}{*}{$\begin{array}{l}\text { Brodmann } \\
\text { area }\end{array}$} & \multirow[t]{2}{*}{ Anatomical structure } & \multirow[t]{2}{*}{$\begin{array}{l}\text { Size } \\
\text { (voxels) }\end{array}$} & \multicolumn{3}{|c|}{$\begin{array}{l}\text { MNI } \\
\text { coordinates }\end{array}$} & \multirow[t]{2}{*}{$\begin{array}{l}t- \\
\text { value }\end{array}$} \\
\hline & & & $x$ & $y$ & $z$ & \\
\hline $\begin{array}{l}9 / 10 / 32 / \\
12\end{array}$ & Medial prefrontal cortex & 4274 & -6 & 20 & -14 & 6.95 \\
\hline $47 / 11$ & Left inferior frontal gyrus & 879 & -48 & 30 & -14 & 6.28 \\
\hline 21 & $\begin{array}{l}\text { Left middle temporal gyrus } \\
\text { Left cerebellum }\end{array}$ & $\begin{array}{l}874 \\
601\end{array}$ & $\begin{array}{l}-62 \\
-28\end{array}$ & $\begin{array}{r}-2 \\
-88\end{array}$ & $\begin{array}{l}-24 \\
-34\end{array}$ & $\begin{array}{l}5.83 \\
5.87\end{array}$ \\
\hline 39 & $\begin{array}{l}\text { Left inferior parietal lobute/ } \\
\text { angular gyrus }\end{array}$ & 489 & -42 & -68 & 28 & 4.42 \\
\hline $47 / 11$ & Right inferior frontal gyrus & 414 & 38 & 44 & -18 & 7.41 \\
\hline \multirow[t]{2}{*}{39} & $\begin{array}{l}\text { Right inferior parietal lobute/ } \\
\text { angular gyrus }\end{array}$ & 363 & 46 & -64 & 36 & 4.20 \\
\hline & Right cerebellum & 347 & 26 & -90 & -32 & 5.76 \\
\hline 21 & Right middle temporal gyrus & 96 & 70 & -32 & -8 & 4.08 \\
\hline 21 & Right middle temporal gyrus & 61 & 64 & -16 & -18 & 3.66 \\
\hline 31 & $\begin{array}{l}\text { Right precuneus/anterior } \\
\text { cingulate cortex }\end{array}$ & 52 & 2 & -60 & 38 & 3.67 \\
\hline
\end{tabular}

Only clusters larger than 50 voxels are listed. 


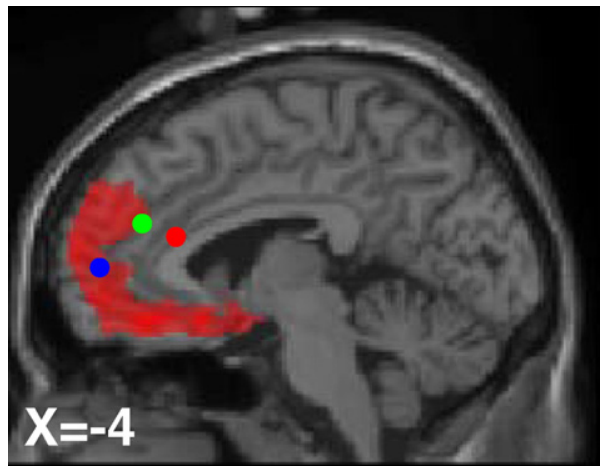

Fig. 4. Dipole locations shown as dots on a saggital slice depicting the midfrontal cluster of BOLD-theta correlations, for the optimal location (red), the nearest voxel showing a significant correlation with theta power (green), and the local maximum in the medial cluster with the lowest amount of unexplained variance (blue).

to the canonical EPI template provided by SPM5 and smoothed with an isotropic Gaussian kernel (FWHM=10 mm).

A linear model was formed by the theta based regressor and the six movement regressors consisting of the realignment parameters provided by the realignment preprocessing step. At the group level, in a random effects analysis, the beta values associated with the theta regressor were tested against zero in a one sample $t$-test. We used a voxel-wise false discovery rate to correct for multiple non-independent comparisons (Benjamini and Hochberg, 1995; Genovese et al., 2002) at the 0.05 level. For the purpose of interpretation we only report clusters that contain at least 50 significant voxels.

\subsection{Dipole fitting}

We compared the brain regions that showed a theta-BOLD correlation with a source location associated with frontal theta based on the following dipole fitting approach. A single dipole was fitted to a grand-average scalp topography using the Fieldtrip software. This grand-average scalp topography was obtained by computing the average over the individual root-mean-square normalized topographies of the selected ICs mixing weights (see Fig. 2). As a head model, we used a standard realistic 3compartment (brain/CSF, skull and skin) boundary element model (BEM) based on the MNI template brain (Oostenveld et al., 2001). In the first approximation the optimal location was found by fitting the dipole at each point of a grid with a resolution of $20 \mathrm{~mm}$. Subsequently, the optimal grid coordinate was used as starting point for a more precise fit using a nonlinear search algorithm.
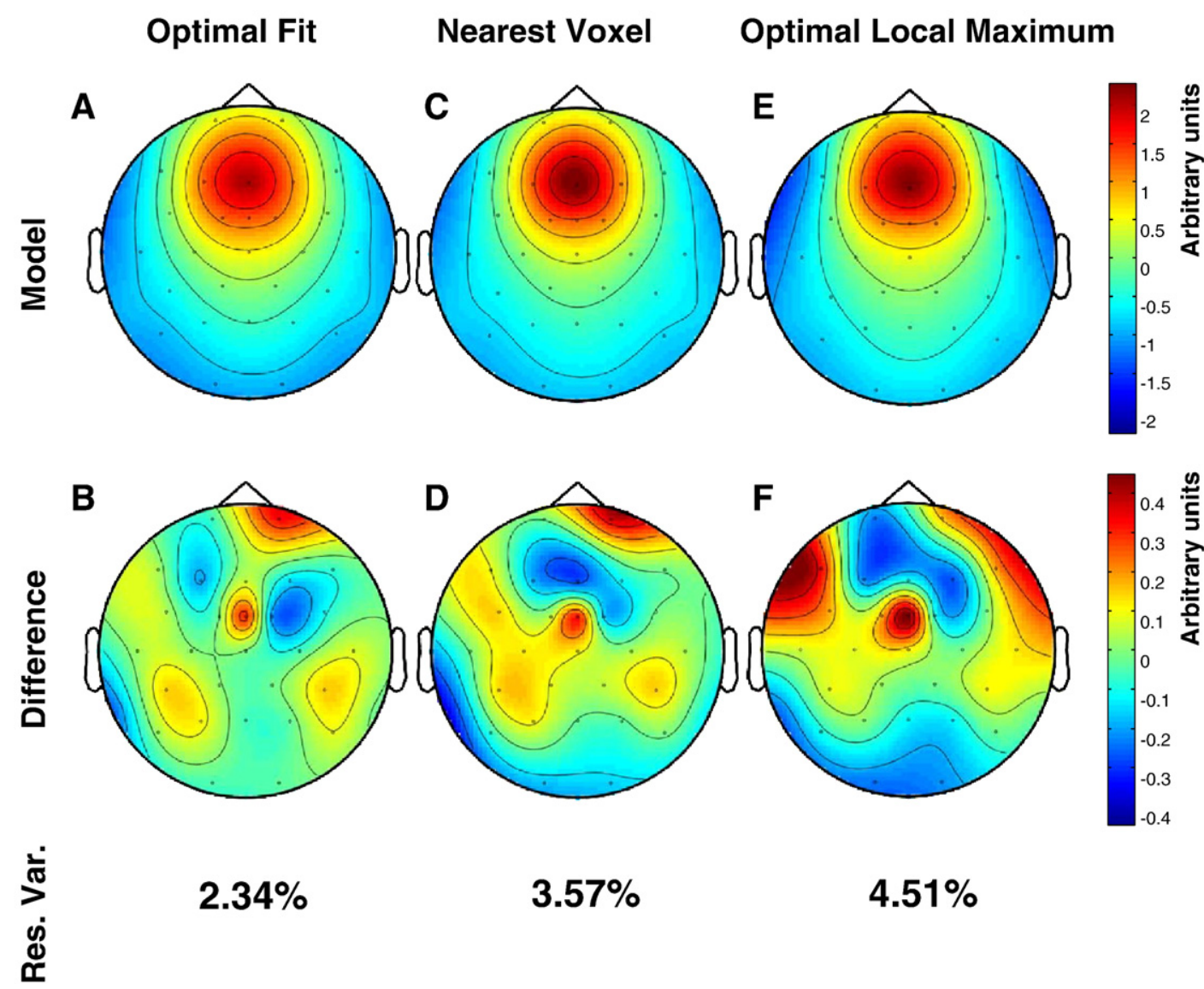

$3.57 \%$

\section{$4.51 \%$}

Fig. 5. Scalp topographies of the modeled part of the grand-average scalp topography of the normalized mixing weights (A, C and E) and the corresponding difference between the model and the grand average (B, D and F). A and B: Dipole explaining maximal amount of variance; C and D: dipole located at the significant voxel closest to the dipole location explaining the most variance; $\mathrm{E}$ and $\mathrm{F}$ : dipole located at the local maximum in the medial frontal cluster that explains the most variance. For the scalp topography to which the dipoles were fitted we refer to Fig. 3A. 
With this unconstrained fit we obtained an optimal dipole location that is located outside the clusters that show a significant BOLD-theta correlation (see Results). To compare this location with possible locations inside the nearest BOLD-theta cluster, two constrained dipole fits were done. For the first constrained fit, the nearest significant voxel was determined. The amplitude and orientation was fitted for a dipole that was fixed at this position. In the second constrained fit, the amplitude and orientation were first fitted separately for each local maximum in the nearest BOLDtheta cluster. Subsequently, the optimal dipole localization that coincides with a local maximum in this BOLD-theta cluster was determined based on the residual variance.

\section{Results}

\subsection{Independent component analysis}

The grand-average topography in Fig. 2A of the normalized mixing weights shows a clear frontal distribution that resembles the frontal theta component described by others (Makeig et al., 2002; Onton et al., 2005). In Fig. 2B the topographies of the selected component for each subject are depicted. Fig. 2C shows the individual and grand-average spectra of the selected components with a clear peak in the theta frequency range at 6.5 Hz. This spectrum is based on the Fourier analysis of $2 \mathrm{~s}$ long Hanning tapered segments, resulting in frequency bins of $0.5 \mathrm{~Hz}$. Note that the time-frequency analysis used in the construction of the regressor is not based on this frequency analysis.

\section{2. fMRI analysis}

No significant positive correlations with fluctuations in theta power were observed. However, a considerable number of brain regions across the cortex and the cerebellum were negatively correlated with frontal theta oscillations (see Fig. 3). A list of negatively correlating regions can be found in Table 1 . This list of regions indicates that in addition to a medial frontal region there is a network of brain regions across the cortex and the cerebellum that correlates with the spontaneous frontal theta power fluctuations observed in resting state.

\subsection{Dipole fitting}

The dipole fitting procedure yielded an optimal dipole location in the anterior cingulate cortex, (residual variance: 2,34\%; MNI coordinates: $-3,29$, 18; see Figs. 4 and 5). This location is just outside the region where the BOLD signal correlates significantly with the theta regressor. The dipole location is approximately $12 \mathrm{~mm}$ from the nearest significant voxel in the medial prefrontal BOLD-theta cluster (MNI coordinates: $-4,40,24)$. A dipole restricted to this location results in a residual variance of $3.57 \%$. The local maximum in the BOLD-theta cluster that explains the most variance of the EEG scalp topography was found at MNI coordinates - 2, 56, 6 (residual variance $4.51 \%$ ), which is $29 \mathrm{~mm}$ from the overall optimal fit.

\section{Discussion}

In the present study, we used simultaneously recorded EEG and fMRI to investigate the BOLD correlates of spontaneous frontal theta power changes, while subjects were quietly lying in the scanner without performing any task. No significant positive correlations between frontal theta power changes and the BOLD signal were observed. Instead, we observed significant negative correlations in medial frontal regions, precuneus/posterior cingulate cortex and bilaterally in the inferior frontal, inferior parietal and middle temporal cortices as well as the cerebellum.

Together, these brain regions constitute the default mode network (DMN) as identified with PET and fMRI (Damoiseaux et al., 2006; Fox et al., 2005; Raichle et al., 2001; Shulman et al., 1997). This is an intrinsically correlated network of brain regions that is regularly observed to deactivate during attentiondemanding cognitive tasks. Activation of this network has recently been linked to stimulus-independent thought, or in other words, mind-wandering (Mason et al., 2007). The presently observed negative correlation of frontal theta power with the DMN therefore suggests that frontal theta activity can be used as an index of DMN activity, at least in the resting state condition.

We envisage three possible accounts for the negative correlation between fluctuations in frontal theta and the BOLD signal in the DMN. The first possibility is that midfrontal theta is not generated in any of the DMN regions. The negative correlations are observed in brain regions that respond with a BOLD-signal decrease to an increase in frontal theta originating from a region where no (significant) BOLD correlation was observed. In this case, the theta-generating process is different from activity in the DMN, although there is a systematic covariation between theta power fluctuations and the DMN. The second possibility is that one (or more) of the observed clusters, most likely the medial frontal region, is the source of mid-frontal theta. Because of the intrinsic correlations within the DMN, the other areas pertaining to this network also show a correlation with theta, although they do not by themselves generate theta-band activity. The third possibility is that the fluctuations in theta amplitude observed on the midfrontal electrodes is a reflection of the fluctuations in theta present in all the clusters observed in this study. If this is the case, then theta-band activity is present throughout DMN, but the frontal theta source is the only part of the network that is readily detectable with scalp-recorded EEG.

There is some evidence supporting the second and third possibilities rather than the first. The first line of evidence is that it has been shown that the medial frontal cortex (our major cluster) is a likely source of frontal theta activity. Source modeling attempts have located the source of frontal theta effects in medial frontal areas (Asada et al., 1999; Gevins et al., 1997; Ishii et al., 1999; Onton et al., 2005). However, most of these attempts used equivalent dipole models and located the frontal theta in or near the anterior cingulate region, somewhat posterior and deeper than the observed medial frontal thetaBOLD cluster (Asada et al., 1999; Gevins et al., 1997; Onton et al., 2005). Also in our dipole modeling attempt the best fit 
was found for a dipole location in the anterior cingulate. However, for a dipole that is placed either at the nearest suprathreshold voxel, or at the local maximum in the medial frontal cluster that explains the most variance, the dipole fit was qualitatively similar to the unconstrained fit. Interestingly, Ishii et al. (1999) used a beamformer approach on MEG data to estimate the location of the theta source, and located it in a region that largely overlaps with the medial frontal $\mathrm{fMRI}$ cluster observed in this experiment. If the cluster is the source location of the frontal theta activity, the substantial size of this cluster suggests a distributed source for frontal theta. The discrepancy observed here between the unconstrained fit and the medial frontal BOLD-theta cluster may be explained by the fact that equivalent dipole models tend to estimate the source location too deep when there is in reality a more superficial distributed source (De Munck et al., 1988).

Another line of evidence suggests that amplitude increases in low frequency oscillations (e.g. delta, theta and alpha) are related to a decreased BOLD signal. Based on theoretical considerations, Kilner et al. (2005) suggest that higher energy dissipation, and therefore a higher BOLD signal, is related to a relative shift in neuronal activity from lower to higher frequencies. This would result in, for instance, reduced delta and theta and increased beta and gamma amplitudes. A relative increase in theta oscillations could therefore lead to a decrease in the BOLD signal. Simultaneous recording of hemodynamic responses and intracortical electrophysiological responses in the visual cortex of cat (Niessing et al., 2005) and monkey (Logothetis et al., 2001; Shmuel et al., 2006) corroborate this notion. In the cat, Niessing et al. (2005) reported negative correlations in between lower frequencies (delta and theta) and the hemodynamic responses, and strong positive correlations with gamma band activity during visual stimulation. Shmuel et al. (2006) found that the negative BOLD response in the monkey primary visual cortex during partial visual field stimulation is accompanied with decreased neural activity in the gamma range $(30-130 \mathrm{~Hz})$. Logothetis et al. (2001) showed that increased gamma band activity during visual stimulation is tightly coupled to the positive BOLD response. In addition, Mizuhara et al. (2004) found predominantly negative relationships between BOLD and frontal theta activity during mental arithmetic. Although, they did not identify these areas as part of the DMN, the pattern is quite similar to that of the present study. In contrast to the latter results, as well as the present results, Sammer et al. (2007) report only positive correlations with theta power. Evidence that an increase in high frequency oscillatory activity results in a BOLD increase in the DMN is provided by Laufs et al. (2003b), who report a positive correlation between beta $(17-23 \mathrm{~Hz})$ amplitude and the BOLD signal in the DMN. The relationship between activity in cortical networks, oscillatory activity and hemodynamic measures in these cases can however be more complex than explained above and more comprehensive spectral EEG information may need to be correlated with hemodynamic measures (Laufs et al., 2006).

More supporting evidence for a direct link between decreased DMN activity and increased frontal theta amplitude lies in the fact that both phenomena are observed in attention-demanding task conditions (for the DMN see Gusnard and Raichle, 2001; Raichle et al., 2001; for frontal theta activity see Burgess and Gruzelier, 1997; Inanaga, 1998; Ishihara and Yoshii, 1972; Laukka et al., 1995; Lazarev, 1998; Smith et al., 1999). It is, therefore, plausible that the inverse relationship also holds in these task conditions.

Above we have presented evidence that suggests a direct link between decreased DMN activity and increased frontal theta amplitude. If this is indeed the case, a common functional interpretation is needed for both phenomena. Currently, the DMN is interpreted as a network of brain regions that is more active when one is not engaged in a cognitively demanding task, while the reverse holds when one is engaged in such tasks. A speculative hypothesis then is that the DMN operates in theta mode (which is reflected in the scalp EEG by an increase in frontal theta) when it becomes less active (i.e., during engagement in a task). This would not only explain the observed BOLD decreases during engagement in a task (Gusnard and Raichle, 2001; Raichle et al., 2001), but it would also explain why frontal theta power has been shown to increase in a wide range of cognitive tasks, such as mental arithmetic (Asada et al., 1999; Burgess and Gruzelier, 1997; Inanaga, 1998; Inouye et al., 1994; Iramina et al., 1996; Ishihara and Yoshii, 1972; Ishii et al., 1999; Lazarev, 1998; Mizuki et al., 1980; Sasaki et al., 1996; Smith et al., 1999), error detection tasks (Luu et al., 2003; Luu et al., 2004), language comprehension tasks (Bastiaansen et al., 2002; Hald et al., 2006) and working memory tasks (Gevins et al., 1997; Jensen and Tesche, 2002; Krause et al., 2000; Onton et al., 2005). However, although this suggestion would account for the observed pattern of theta power increases, it appears to contradict several previous functional interpretations that increased frontal theta oscillations reflect synchronous activity in brain regions that are involved in cognitively demanding tasks (Inanaga, 1998; Ishihara and Yoshii, 1972; Jensen and Tesche, 2002; Laukka et al., 1995; Onton et al., 2005; Smith et al., 1999). We feel that more experimental work is needed in order to differentiate between these two opposing possibilities.

The present study has focused on fluctuations in frontal theta power during resting state. There are also many other brain regions that display oscillatory dynamics in the theta frequency range (for a review see Kahana et al., 2001). It is possible that theta-band activity in different brain regions is associated with different types of neural and cognitive processes. In addition, the sign of the correlation between theta power and BOLD might differ between brain regions. For instance, in intracranial recordings in humans, Canolty et al. (2006) found a strong modulation of theta activity on gamma oscillations, but also reported a positive amplitude correlation between the two rhythms. Since gamma is demonstrated to correlate positively with BOLD (e.g. Logothetis et al., 2001; Niessing et al., 2005), and theta seems to correlate negatively with BOLD (Mizuhara et al., 2004; Niessing et al., 2005; present study), it is difficult to predict the sign of the correlation between BOLD and theta or gamma. This illustrates that more insight is needed in how different oscillatory signals from different brain regions relate to the hemodynamic measures obtained by fMRI and PET. This will be crucial for linking the 
knowledge about cognitive processes obtained with electrophysiological and hemodynamic research methods.

\section{Acknowledgements}

This work was supported by a grant from the Netherlands Organisation for Scientific Research (NWO, grant number 40003-277). We would like to thank Paul Gaalman and Erik van den Boogert for technical assistance and Helmut Laufs for helpful comments on an earlier version of the manuscript.

\section{References}

Aghakhani, Y., Bagshaw, A.P., Benar, C.G., Hawco, C., Andermann, F., Dubeau, F., Gotman, J., 2004. fMRI activation during spike and wave discharges in idiopathic generalized epilepsy. Brain 127, 1127-1144.

Al-Asmi, A., Benar, C.G., Gross, D.W., Khani, Y.A., Andermann, F., Pike, B., Dubeau, F., Gotman, J., 2003. fMRI activation in continuous and spiketriggered EEG-fMRI studies of epileptic spikes. Epilepsia 44, 1328-1339.

Allen, P.J., Polizzi, G., Krakow, K., Fish, D.R., Lemieux, L., 1998. Identification of EEG events in the MR scanner: the problem of pulse artifact and a method for its subtraction. Neuroimage 8, 229-239.

Allen, P.J., Josephs, O., Turner, R., 2000. A method for removing imaging artifact from continuous EEG recorded during functional MRI. Neuroimage $12,230-239$.

Asada, H., Fukuda, Y., Tsunoda, S., Yamaguchi, M., Tonoike, M., 1999. Frontal midline theta rhythms reflect alternative activation of prefrontal cortex and anterior cingulate cortex in humans. Neurosci. Lett. 274, 29-32.

Bastiaansen, M.C.M., van Berkum, J.J.A., Hagoort, P., 2002. Syntactic processing modulates the theta rhythm of the human EEG. Neuroimage $17,1479-1492$.

Bell, A., Sejnowski, T., 1995. An information-maximization approach to blind separation and blind deconvolution. Neural. Comput. 7, 1129-1159.

Benar, C.G., Grova, C., Kobayashi, E., Bagshaw, A.P., Aghakhani, Y., Dubeau, F.O., Gotman, J., 2006. EEG-fMRI of epileptic spikes: concordance with EEG source localization and intracranial EEG. Neuroimage 30, 1161-1170.

Benjamini, Y., Hochberg, Y., 1995. Controlling the false discovery rate - a practical and powerful approach to multiple testing. J. R. Stat. Soc., Ser. B. Stat. Methodol 57, 289-300.

Bonmassar, G., Anami, K., Ives, J., Belliveau, J.W., 1999. Visual evoked potential (VEP) measured by simultaneous 64-channel EEG and 3T fMRI. Neuroreport 10, 1893-1897.

Bonmassar, G., Purdon, P.L., Jaaskelainen, I.P., Chiappa, K., Solo, V., Brown, E.N., Belliveau, J.W., 2002. Motion and ballistocardiogram artifact removal for interleaved recording of EEG and EPs during MRI. Neuroimage 16, 1127-1141.

Burgess, A.P., Gruzelier, J.H., 1997. Short duration synchronization of human theta rhythm during recognition memory. Neuroreport 8, 1039-1042.

Canolty, R.T., Edwards, E., Dalal, S.S., Soltani, M., Nagarajan, S.S., Kirsch, H.E., Berger, M.S., Barbaro, N.M., Knight, R.T., 2006. High gamma power is phaselocked to theta oscillations in human neocortex. Science 313, 1626-1628.

Damoiseaux, J.S., Rombouts, S.A.R.B., Barkhof, F., Scheltens, P., Stam, C.J., Smith, S.M., Beckmann, C.F., 2006. Consistent resting-state networks across healthy subjects. Proc. Natl. Acad. Sci.U. S. A. 103, 13848-13853.

De Munck, J.C., Van Dijk, B.W., Spekreijse, H., 1988. Mathematical dipoles are adequate to describe realistic generators of human brain activity. IEEE Trans. Biomed. Eng. 35, 960-966.

Debener, S., Ullsperger, M., Siegel, M., Fiehler, K., von Cramon, D.Y., Engel, A.K., 2005. Trial-by-trial coupling of concurrent electroencephalogram and functional magnetic resonance imaging identifies the dynamics of performance monitoring. J. Neurosci. 25, 11730-11737.

Debener, S., Ullsperger, M., Siegel, M., Engel, A.K., 2006. Single-trial EEG-fMRI reveals the dynamics of cognitive function. Trends Cogn. Sci. 10, 558-563.

Delorme, A., Makeig, S., 2004. EEGLAB: an open source toolbox for analysis of single-trial EEG dynamics including independent component analysis. J. Neurosci. Methods 134, 9-21.
Eichele, T., Specht, K., Moosmann, M., Jongsma, M.L.A., Quiroga, R.Q., Nordby, H., Hugdahl, K., 2005. Assessing the spatiotemporal evolution of neuronal activation with single-trial event-related potentials and functional MRI. Proc. Natl. Acad. Sci.U. S. A. 102, 17798-17803.

Feige, B., Scheffler, K., Esposito, F., Di Salle, F., Hennig, J., Seifritz, E., 2005. Cortical and subcortical correlates of electroencephalographic alpha rhythm modulation. J. Neurophysiol. 93, 2864-2872.

Fox, M.D., Snyder, A.Z., Vincent, J.L., Corbetta, M., Van Essen, D.C., Raichle, M.E., 2005. The human brain is intrinsically organized into dynamic, anticorrelated functional networks. Proc. Natl. Acad. Sci.U. S. A. 102, 9673-9678.

Genovese, C.R., Lazar, N.A., Nichols, T., 2002. Thresholding of statistical maps in functional neuroimaging using the false discovery rate. Neuroimage 15 , 870-878.

Gevins, A., Smith, M.E., McEvoy, L., Yu, D., 1997. High-resolution EEG mapping of cortical activation related to working memory: effects of task difficulty, type of processing, and practice. Cereb. Cortex 7, 374-385.

Goldman, R.I., Stern, J.M., Engel, J., Cohen, M.S., 2002. Simultaneous EEG and fMRI of the alpha rhythm. Neuroreport 13, 2487-2492.

Goncalves, S.I., de Munck, J.C., Pouwels, P.J.W., Schoonhoven, R., Kuijer, J.P.A., Maurits, N.M., Hoogduin, J.M., Van Someren, E.J.W., Heethaar, R.M., da Silva, F.H.L., 2006. Correlating the alpha rhythm to BOLD using simultaneous EEG/fMRI: inter-subject variability. Neuroimage 30, 203-213.

Gusnard, D.A., Raichle, M.E., 2001. Searching for a baseline: functional imaging and the resting human brain. Nat. Rev. Neurosci. 2, 685-694.

Hald, L.A., Bastiaansen, M.C.M., Hagoort, P., 2006. EEG theta and gamma responses to semantic violations in online sentence processing. Brain Lang. 96, 90-105.

Inanaga, K., 1998. Frontal midline theta rhythm and mental activity. Psychiatry. Clin. Neurosci. 52, 555-566.

Inouye, T., Shinosaki, K., Iyama, A., Matsumoto, Y., Toi, S., Ishihara, T., 1994. Potential flow of frontal midline theta-activity during a mental task in the human electroencephalogram. Neurosci. Lett. 169, 145-148.

Iramina, K., Ueno, S., Matsuoka, S., 1996. MEG and EEG topography of frontal midline theta rhythm and source localization. Brain Topogr. 8, 329-331.

Ishihara, T., Yoshii, N., 1972. Multivariate analytic study of EEG and mental activity in juvenile delinquents. Electroencephalogr. Clin. Neurophysiol. 33, $71-80$.

Ishii, R., Shinosaki, K., Ukai, S., Inouye, T., Ishihara, T., Yoshimine, T., Hirabuki, N., Asada, H., Kihara, T., Robinson, S.E., Takeda, M., 1999. Medial prefrontal cortex generates frontal midline theta rhythm. Neuroreport $10,675-679$.

Jensen, O., Tesche, C.D., 2002. Frontal theta activity in humans increases with memory load in a working memory task. Eur. J. Neurosci. 15, 1395-1399.

Kahana, M.J., Seelig, D., Madsen, J.R., 2001. Theta returns. Curr. Opin. Neurobiol. 11 (6), 739-744.

Kilner, J.M., Mattout, J., Henson, R., Friston, K.J., 2005. Hemodynamic correlates of EEG: a heuristic. Neuroimage 28, 280-286.

Krause, C.M., Sillanmaki, L., Koivisto, M., Saarela, C., Haggqvist, A., Laine, M., Hamalainen, H., 2000. The effects of memory load on event-related EEG desynchronization and synchronization. Clin. Neurophysiol. 111, 2071-2078.

Laufs, H., Kleinschmidt, A., Beyerle, A., Eger, E., Salek-Haddadi, A., Preibisch, C., Krakow, K., 2003a. EEG-correlated fMRI of human alpha activity. Neuroimage 19, 1463-1476.

Laufs, H., Krakow, K., Sterzer, P., Eger, E., Beyerle, A., Salek-Haddadi, A., Kleinschmidt, A., 2003b. Electroencephalographic signatures of attentional and cognitive default modes in spontaneous brain activity fluctuations at rest. Proc. Natl. Acad. Sci.U. S. A. 100, 11053-11058.

Laufs, H., Holt, J.L., Elfont, R., Krams, M., Paul, J.S., Krakow, K., Kleinschmidt, A., 2006. Where the BOLD signal goes when alpha EEG leaves. Neuroimage 31, 1408-1418.

Laukka, S.J., Jarvilehto, T., Alexandrov, Y.I., Lindqvist, J., 1995. Frontal midline-theta related to learning in a simulated driving task. Biol. Psychol. 40, 313-320.

Lazarev, V.V., 1998. On the intercorrelation of some frequency and amplitude parameters of the human EEG and its functional significance. Communication I: multidimensional neurodynamic organization of functional states of the brain during intellectual, perceptive and motor activity in normal subjects. Int. J. Psychophysiol. 28, 77-98. 
Lemieux, L., Salek-Haddadi, A., Josephs, O., Allen, P., Toms, N., Scott, C. Krakow, K., Turner, R., Fish, D.R., 2001. Event-related fMRI with simultaneous and continuous EEG: description of the method and initial case report. Neuroimage 14, 780-787.

Logothetis, N.K., Pauls, J., Augath, M., Trinath, T., Oeltermann, A., 2001. Neurophysiological investigation of the basis of the fMRI signal. Nature $412,150-157$.

Luu, P., Tucker, D.M., Derryberry, D., Reed, M., Poulsen, C., 2003. Electrophysiological responses to errors and feedback in the process of action regulation. Psychol. Sci. 14, 47-53.

Luu, P., Tucker, D.M., Makeig, S., 2004. Frontal midline theta and the errorrelated negativity: neurophysiological mechanisms of action regulation. Clin. Neurophysiol. 115, 1821-1835.

Makeig, S., Jung, T.P., Bell, A.J., Ghahremani, D., Sejnowski, T.J., 1997. Blind separation of auditory event-related brain responses into independent components. Proc. Natl. Acad. Sci.U. S. A. 94, 10979-10984.

Makeig, S., Westerfield, M., Jung, T.P., Enghoff, S., Townsend, J., Courchesne, E., Sejnowski, T.J., 2002. Dynamic brain sources of visual evoked responses. Science 295, 690-694.

Mason, M.F., Norton, M.I., Van Horn, J.D., Wegner, D.M., Grafton, S.T., Macrae, C.N., 2007. Wandering minds: the default network and stimulusindependent thought. Science 315, 393-395.

Mitra, P.P., Pesaran, B., 1999. Analysis of dynamic brain imaging data. Biophys. J. 76, 691-708.

Mizuhara, H., Wang, L.Q., Kobayashi, K., Yamaguchi, Y., 2004. A long-range cortical network emerging with theta oscillation in a mental task Neuroreport 15, 1233-1238.

Mizuki, Y., Tanaka, M., Isozaki, H., Nishijima, H., Inanaga, K., 1980. Periodic appearance of theta rhythm in the frontal midline area during performance of a mental task. Electroencephalogr. Clin. Neurophysiol. 49, 345-351.

Moosmann, M., Ritter, P., Krastel, I., Brink, A., Thees, S., Blankenburg, F., Taskin, B., Obrig, H., Villringer, A., 2003. Correlates of alpha rhythm in functional magnetic resonance imaging and near infrared spectroscopy. Neuroimage 20, 145-158
Niessing, J., Ebisch, B., Schmidt, K.E., Niessing, M., Singer, W., Galuske, R.A., 2005. Hemodynamic signals correlate tightly with synchronized gamma oscillations. Science 309, 948-951.

Onton, J., Delorme, A., Makeig, S., 2005. Frontal midline EEG dynamics during working memory. Neuroimage 27, 341-356.

Oostenveld, R., Praamstra, P., Stegeman, D.F., van Oosterom, A., 2001. Overlap of attention and movement-related activity in lateralized event-related brain potentials. Clin. Neurophysiol. 112, 477-484.

Raichle, M.E., MacLeod, A.M., Snyder, A.Z., Powers, W.J., Gusnard, D.A., Shulman, G.L., 2001. A default mode of brain function. Proc. Natl. Acad. Sci.U. S. A. 98, 676-682.

Salek-Haddadi, A., Merschhemke, M., Lemieux, L., Fish, D.R., 2002. Simultaneous EEG-correlated ictal fMRI. Neuroimage 16, 32-40.

Sammer, G., Blecker, C., Gebhardt, H., Kirsch, P., Stark, R., Vaitl, D., 2005. Acquisition of typical EEG waveforms during fMRI: SSVEP, LRP, and frontal theta. Neuroimage 24, 1012-1024.

Sammer, G., Blecker, C., Gebhardt, H., Bisschoff, M., Stark, R., Morgen, K., Vaitl, D., 2007. Relationship between regional hemodynamic activity and simultaneously recorded EEG-theta associated with mental arithmeticinduced workload. Hum. Brain. Mapp. 28, 793-803.

Sasaki, K., Tsujimoto, T., Nishikawa, S., Nishitani, N., Ishihara, T., 1996. Frontal mental theta wave recorded simultaneously with magnetoencephalography and electroencephalography. Neurosci. Res. 26, 79-81.

Shmuel, A., Augath, M., Oeltermann, A., Logothetis, N.K., 2006. Negative functional MRI response correlates with decreases in neuronal activity in monkey visual area V1. Nat. Neurosci. 9, 569-577.

Shulman, G.L., Fiez, J.A., Corbetta, M., Buckner, R.L., Miezin, F.M., Raichle, M.E., Petersen, S.E., 1997. Common blood flow changes across visual tasks. 2. Decreases in cerebral cortex. J. Cogn. Neurosci. 9, 648-663.

Smith, M.E., McEvoy, L.K., Gevins, A., 1999. Neurophysiological indices of strategy development and skill acquisition. Brain. Res. Cogn. Brain. Res. 7, 389-404.

Srinivasan, R., Winter, W.R., Nunez, P.L., 2006. Source analysis of EEG oscillations using high-resolution EEG and MEG. Prog. Brain Res. 159, 29-42. 